Boletín de la Sociedad Zoológica del Uruguay, 2021

Vol. 30 (2): e30.2.9

ISSN 2393-6940

https://journal.szu.org.uy

DOI: https://doi.org/10.26462/30.2.9

\title{
CARACTERIZACIÓN ESTACIONAL DE LA DIETA Y PERFIL DE ÁCIDOS GRASOS DE JUVENILES DE Paralichthys orbignyanus (Jenyns, 1842)
}

\author{
Larisa Magnone ${ }^{(D)}$, Juan Gadea (D) , María Salhi (D) , Erika Nuñez (D), \\ Martin Bessonart* (DD .
}

Universidad de la República, Facultad de Ciencias, Instituto de Ecología y Ciencias Ambientales, Laboratorio de Recursos Naturales, Iguá 4225, 11400 Montevideo, Uruguay.

*Autor para correspondencia: martinb@fcien.edu.uy

Fecha de recepción: 20 de julio de 2021

Fecha de aceptación: 27 de octubre de 2021

\section{RESUMEN}

Los estuarios se caracterizan por tener alta disponibilidad de alimento y refugio, constituyendo áreas de cría para muchas especies acuáticas. En ellos también ocurre variabilidad estacional en la disponibilidad de presas. Conocer la dieta y su variación temporal aporta a la comprensión de las relaciones tróficas y del rol de las especies. Los ácidos grasos (AG) constituyen una aproximación para detectar variaciones temporales de dieta, pues las especies reflejan en sus AG los AG de sus presas. Los objetivos del trabajo fueron analizar la variación temporal del perfil de $A G$ de las reservas energéticas de juveniles de Paralichthys orbignyanus y caracterizar su dieta, así como la variación estacional durante un ciclo anual mediante análisis de contenido estomacal. Se colectaron 78 juveniles en el arroyo Valizas entre marzo y noviembre de 2012. La dieta estuvo compuesta mayoritariamente por poliquetos $y$ misidáceos, y el índice de importancia relativa no mostró diferencias significativas a lo largo del año $(p>0,05)$. Sin embargo, en el análisis de correspondencia los poliquetos fueron dominantes en mayo, y en noviembre predominaron los misidáceos. El perfil de ácidos grasos de los peces presentó una segregación temporal en la mayoría de las campañas, reflejando las variaciones estacionales de la dieta.

Palabras clave: lenguados, estuarios, contenido estomacal, ácidos grasos.

\section{ABSTRACT}

Seasonal characterization of the diet and fatty acids profiles of juvenile Paralichthys orbignyanus (Jenyns, 1842). The refuge and high food availability found in estuaries turn them into nursery areas for many aquatic animals. In such environments, seasonal variation of prey availability also occurs. Knowledge on species diet composition and their temporal variations, contributes to the understanding of trophic interactions and the role that the species play in the ecosystem. Fatty acids (FA) serve as an approximation to detect temporal variation of the diet, since consumers FA reflect their prey FA. The objectives of this study were the analysis of the temporal variation of storage lipids' FA profiles of juvenile Paralichthys orbignyanus, and the characterization of their diet and its seasonal variation throughout an annual cycle assessed by stomach contents. Juvenile flatfish $(n=78)$ were collected in the Valizas stream from March to November 2012. Diet of $P$. orbignyanus was mainly composed of polychaetes and misidiaceans, and the relative importance index did not varied significantly throughout the year $(p>0.05)$. However, a correspondence analysis showed a dominance of polychaetes consumption in May and misidiaceans in November. A temporal diet segregation in almost all seasons was observed through the analysis of fish fatty acid profiles, reflecting the seasonal variations of the diet.

Key words: flatfishes, estuaries, stomach contents, fatty acids.

\section{INTRODUCCIÓN}

Los estuarios son ambientes altamente productivos que constituyen áreas de cría para muchas especies acuáticas (McLusky y Elliott, 2004a; Cowan, Yañez-Arancibia, Sánchez-Gil y Deegan, 2013). Podrían considerarse como hábitats de alta calidad por su gran disponibilidad de alimento y zonas 


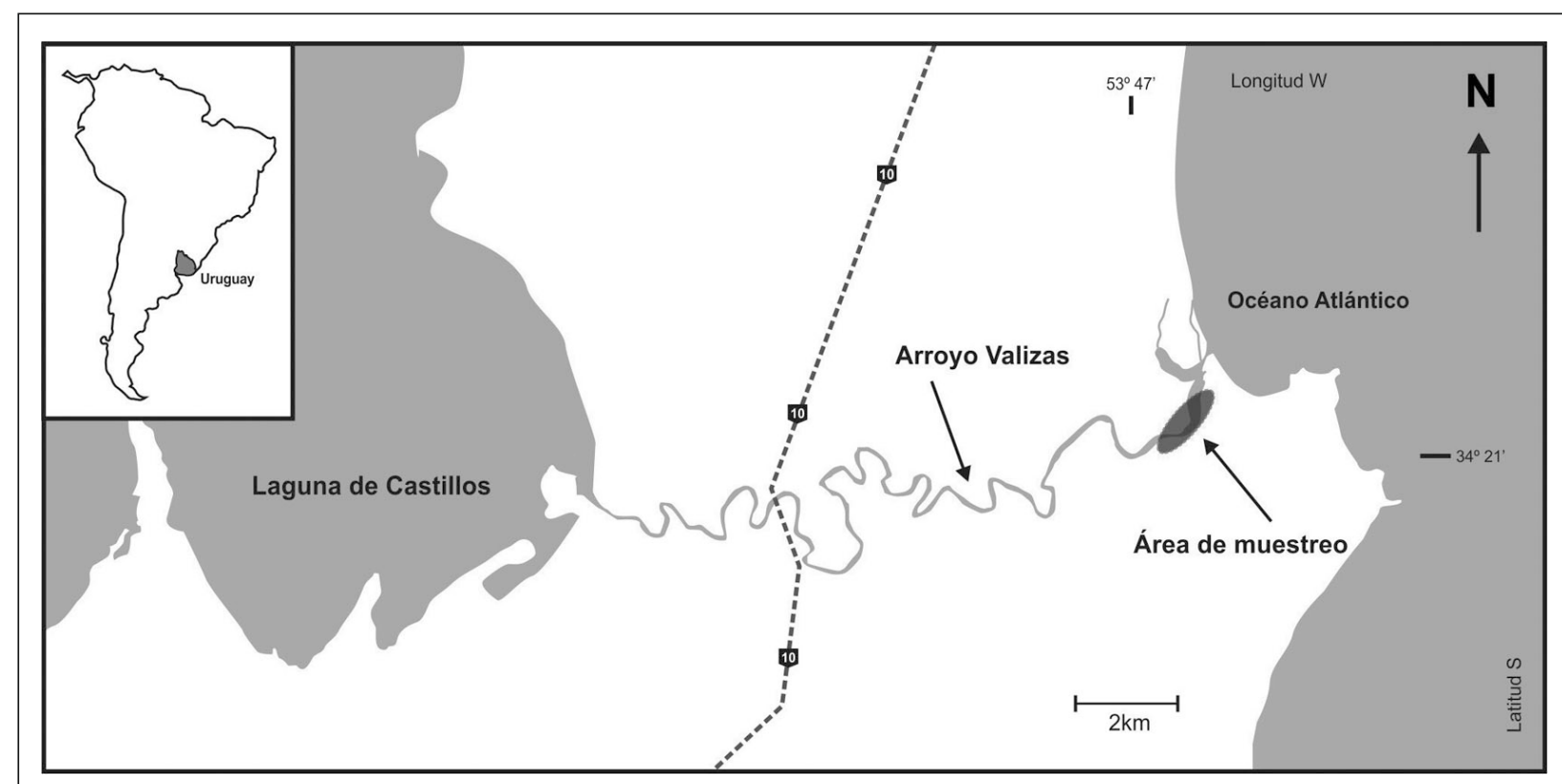

Fig. 1. Sitio de muestreo para captura de juveniles de Paralichthys orbignyanus en el arroyo Valizas, Uruguay.

de refugio, lo cual aumenta la sobrevivencia y el crecimiento de muchas especies de peces en estado juvenil (Gibson, 1994; Peterson, 2003; Wouters y Cabral, 2009). En ellos se ha reportado variabilidad estacional en la disponibilidad de presas en organismos que predan sobre invertebrados bentónicos (Curtis, Galbraith, Smyth y Thompson, 1985; McLusky y Elliott, 2004b) así como también organismos del zooplancton (Froneman, 2001; Espinosa, 2017).

Paralichthys orbignyanus es un pez plano muy común en las costas uruguayas, que se distribuye desde Río de Janeiro hasta el Golfo de San Matías (Cazorla, 2005). Es una especie eurihalina, estuarinadependiente que se adentra en los estuarios y en las lagunas costeras que se comunican con el mar (VazFerreira, 1969). Sus juveniles son habitualmente encontrados en zonas estuarinas (Rivera, García de la Rosa y Díaz de Astarloa, 2001; Acuña, Passadore y Gimenez, 2010; Magnone et al., 2015a).

Los peces planos o lenguados (Pleuronectiformes) son un componente ecológicamente destacado de estos ecosistemas, ya que constituyen una vía energética de conversión de la producción bentónica hacia niveles superiores en las redes tróficas (Elliot et al., 2002; Link, Fogarty y Langton, 2005). El conocimiento acerca de la dieta de los peces, así como su variación en el tiempo aporta a la comprensión de las relaciones tróficas y del rol que cumplen dichas especies dentro los ambientes (Able y Grothues, 2007).

Los lenguados comúnmente se alimentan de manera oportunista, consumiendo las presas más abundantes en el ambiente, mostrando preferencias por dos grupos de presas (peces y camarones y/o poliquetos y pequeños crustáceos) según su tamaño (Link et al., 2005). A su vez, la comunidad bentónica (ej: crustáceos y poliquetos) en zonas estuarinas varía ampliamente, reflejándose en la composición de la dieta de estos peces (Williams y Williams, 1998; Link et al., 2005). La información acerca de los hábitos alimenticios de la mayoría de las especies proviene de métodos como el análisis del contenido estomacal (Hyslop, 1980). En diferentes estudios sobre varias especies de pleuronectiformes, se ha observado que los poliquetos y los anfípodos representan entre el 40 y el $70 \%$ de la dieta de los juveniles (Piet, Pfisterer y Rijnsdop, 1999; Hostens y Mees, 1999; Rivera, García de la Rosa y Díaz de Astarloa, 2001). Dicho hábito usualmente cambia con su desarrollo ontogenético convirtiéndose en piscívoros (Garrison y Link, 2000). A su vez esta variación se puede ver reflejada en los organismos a través de la composición de ácidos grasos de los tejidos de reserva, ya que las especies suelen reflejar allí la composición de ácidos grasos de sus presas (Sargent, Tocher y Bell, 2002). De esta forma, el análisis de la variación de los perfiles de ácidos grasos de una especie durante su desarrollo nos permite detectar posibles cambios en la dieta en un período de tiempo (Iverson, 2009).

Los objetivos de este trabajo fueron analizar la variación temporal del perfil de ácidos grasos de las reservas energéticas de juveniles de Paralichthys orbignyanus y caracterizar su dieta así como la variación estacional durante un ciclo anual mediante análisis de contenido estomacal. 


\section{MATERIALES Y MÉTODOS}

Área de estudio. El trabajo se realizó en el arroyo Valizas (Fig. 1) (Rocha, Uruguay), un canal fluvial de alta sinuosidad de régimen estuarino, de unos $15 \mathrm{~km}$ de extensión, que funciona como canal de comunicación entre la Laguna de Castillos y el océano Atlántico, con el que se conecta intermitentemente a través de una barra arenosa.

Captura y manejo de ejemplares. Se realizaron cinco muestreos para colecta de ejemplares juveniles del lenguado $P$. orbignyanus (marzo, mayo, julio, setiembre y noviembre de 2012). Los individuos juveniles fueron capturados mediante encierros con red de arrastre manual ( $1 \mathrm{~mm}$ de abertura de malla, 10 $\mathrm{m}$ de longitud y $1 \mathrm{~m}$ de alto). Se realizaron nueve encierros por campaña. Los individuos colectados fueron eutanasiados por sobredosis de anestésico (Benzocaína 4 ppm), medidos (LT) y pesados (0.01 g). Para los análisis de ácidos grasos y contenido estomacal se colectó músculo dorsal con piel y el estómago de todos los individuos. El músculo fue conservado en frío $\left(-20^{\circ} \mathrm{C}\right)$ para su posterior análisis y el contenido estomacal fue analizado en fresco bajo lupa. Se registró el número de estómagos vacíos.

Análisis de contenido estomacal. Los ítems presas encontrados en los estómagos se identificaron al nivel taxonómico más bajo posible, se contabilizaron y se pesaron en fresco para cada estómago colectado. Para la descripción de la importancia relativa de cada ítem presa en la dieta de $P$. orbignyanus en las diferentes campañas se utilizó el índice de importancia relativa (IRI) (Pinkas, Oliphanty Iverson, 1971). IRI $=(\% \mathrm{~N}+\% \mathrm{~W}) * \% \mathrm{FO}$, donde $\% \mathrm{~N}$ (abundancia relativa): representa el número de ítems presa i en relación al total de muestras analizadas. $\% \mathrm{~N}=\left(\mathrm{N}^{\circ}\right.$ de ítem presa $\mathrm{i} / \mathrm{N}^{\circ}$ total de ítem presas ingeridas) $\mathrm{x} 100$. $\% \mathrm{~W}$ (biomasa relativa): es el porcentaje de biomasa relativa de cada ítem presa. $\% \mathrm{~W}=$ (peso del ítem presa i / peso total de los ítems presa) x 100. \%FO (porcentaje de frecuencia de ocurrencia): representa la presencia o ausencia del ítem presa i en los estómagos de la muestra. $\% \mathrm{FO}=\left(\mathrm{N}^{\circ}\right.$ de estómagos en que aparece un ítem presa $/ \mathrm{N}^{\circ}$ total de estómagos con contenido) x 100. Luego los IRI fueron estandarizados al $100 \%$ (\%IRI) donde $0 \%$ (ítem ausente) y $100 \%$ (único ítem consumido) (Cazorla y Forte, 2005). El índice de vacuidad IV representa el porcentaje de estómagos vacíos y fue calculado como $\mathrm{IV}=\left(\mathrm{N}^{\circ}\right.$ de estómagos vacíos $/ \mathrm{N}^{\circ}$ total de estómagos analizados) $\mathrm{x}$ 100 (Molinero y Flos, 1992). Se utilizó el diagrama de Amundsen et al. (1996) para evaluar la importancia de los diferentes ítems presa en la dieta de $P$. orbignyanus. Este análisis grafica la frecuencia de ocurrencia (FO) frente a la abundancia específica (P) de cada ítem presa. Abundancia específica $(P)$ de cada presa: representa la abundancia del ítem presa $x$ en los estómagos de la muestra donde el ítem presa $x$ se encuentra presente. $\mathrm{P}=\left(\boldsymbol{\Sigma}\right.$ ítem $\mathrm{x} / \mathrm{N}^{\circ}$ total de presas presentes en los estómagos con ítem $\mathrm{x}$ ) $\mathrm{x} 100$.

Índices de condición. El factor de condición (FC) que relaciona el peso y la longitud de los animales es a menudo utilizado para cuantificar el estado de salud de los peces, pudiendo asociarse al estado de delgadez 0 gordura de los mismos (Ricker, 1975). $\mathrm{FC}=\left(\operatorname{Peso}(\mathrm{g}) /[\mathrm{LT}(\mathrm{cm})]^{3}\right) \times 100$. Otra aproximación complementaria a la anterior comúnmente utilizada es la cuantificación del contenido o tenor lipídico en sus tejidos de reserva (\%Líp. T) (Arts y Kohler, 2009).

Extracción y separación de lípidos y análisis de ácidos grasos. El músculo con piel de cinco ejemplares por campaña fue liofilizado y homogeneizado previo a su análisis. La extracción y cuantificación de los lípidos totales se realizó de acuerdo a la metodología propuesta por Folch, Lees y Stanley (1957). Debido a que los lípidos neutros reflejan mejor la composición dietaria (Sargent et al., 2002), esta fracción fue separada por cromatografía de adsorción en columna según Juaneda y Rocquelin (1985). Los ésteres metílicos de ácidos grasos (FAMES) de los lípidos neutros se obtuvieron por transesterificación en solución de metanol con ácido sulfúrico según Christie y Han (2010). Los FAMES fueron separados e identificados por cromatografía gaseosa por medio de la comparación de los tiempos de retención de estándares (Salhi y Bessonart, 2013). Los ácidos grasos fueron cuantificados como porcentaje del área total de ácidos grasos identificados.

Análisis de datos. Para comparar el tenor lipídico (\%Líp. T), así como el factor de condición (FC) entre las diferentes campañas se utilizó un análisis de varianza de una vía (Past, 3.0). Con el fin de analizar la variación temporal de la dieta, en base al \%IRI, se utilizó el test no paramétrico de F Snedecor (F) (Past, 3.16) debido a que los datos no presentaron distribución normal pero sí homogeneidad de varianza. Para estudiar la variación de la dieta entre las diferentes campañas de muestreo se realizó un análisis de correspondencia (CA) (Past, 3.16). Este análisis incluyó sólo aquellos ítems presa con una frecuencia de ocurrencia superior al $20 \%$ en al menos uno de los meses estudiados (Masdeu, Teixeira de Mello, Loureiro y Arim, 2011). Se realizó un análisis exploratorio de los perfiles de ácidos grasos mediante un agrupamiento jerárquico utilizando la distancia euclideana y todos los AG reconocidos (Hierachical clustering analysis, Past 3.0). 
Tabla 1. Contenido estomacal de los especímenes capturados, expresados como Índice de importancia relativa estandarizado (\% IRI). Índice de Vacuidad (IV) para la totalidad de los individuos colectados discriminados por campaña y su unificación (campaña I a V).

\begin{tabular}{|c|c|c|c|c|c|c|}
\hline & $\begin{array}{l}\text { Campaña I (mar) } \\
\text { \%IRI }\end{array}$ & $\begin{array}{c}\text { Campaña II (may) } \\
\% I R I\end{array}$ & $\begin{array}{c}\text { Campaña III (jul) } \\
\% I R I\end{array}$ & $\begin{array}{c}\text { Campaña IV (set) } \\
\text { \%IRI }\end{array}$ & $\begin{array}{c}\text { Campaña V (nov) } \\
\% \text { IRI }\end{array}$ & $\begin{array}{c}\text { Campañas I-V (Total) } \\
\% \text { IRI }\end{array}$ \\
\hline \multicolumn{7}{|l|}{ Vertebrados } \\
\hline Peces óseos & 0.00 & 0.00 & 0.00 & 33.48 & 0.37 & 3.14 \\
\hline \multicolumn{7}{|l|}{ Invertebrados } \\
\hline \multicolumn{7}{|l|}{ Crustáceos } \\
\hline Palaemonetes argentinus & 0.00 & 0.00 & 18.24 & 53.50 & 0.81 & 9.60 \\
\hline Neomysis americana & 47.87 & 0.11 & 81.76 & 6.67 & 91.32 & 36.66 \\
\hline Cyptograpsus angulatus & 0.00 & 0.06 & 0.00 & 0.49 & 4.99 & 0.90 \\
\hline Isopoda & 0.00 & 0.02 & 0.00 & 0.00 & 0.00 & 0.01 \\
\hline Amphipoda & 0.00 & 0.00 & 0.00 & 0.00 & 0.10 & 0.01 \\
\hline \multicolumn{7}{|l|}{ Anélidos } \\
\hline Polychaeta & 52.13 & 99.81 & 0.00 & 5.54 & 1.57 & 49.53 \\
\hline \multicolumn{7}{|l|}{ Moluscos } \\
\hline Heleobia australis & 0.00 & 0.00 & 0.00 & 0.33 & 0.85 & 0.16 \\
\hline $\mathrm{N}^{\circ}$ de muestras & 5 & 31 & 13 & 16 & 13 & 78 \\
\hline $\mathrm{N}^{\circ}$ de items presas & 2 & 4 & 2 & 6 & 7 & 8 \\
\hline IV & 20.0 & 16.1 & 30.8 & 6.3 & 15.4 & 16.7 \\
\hline
\end{tabular}

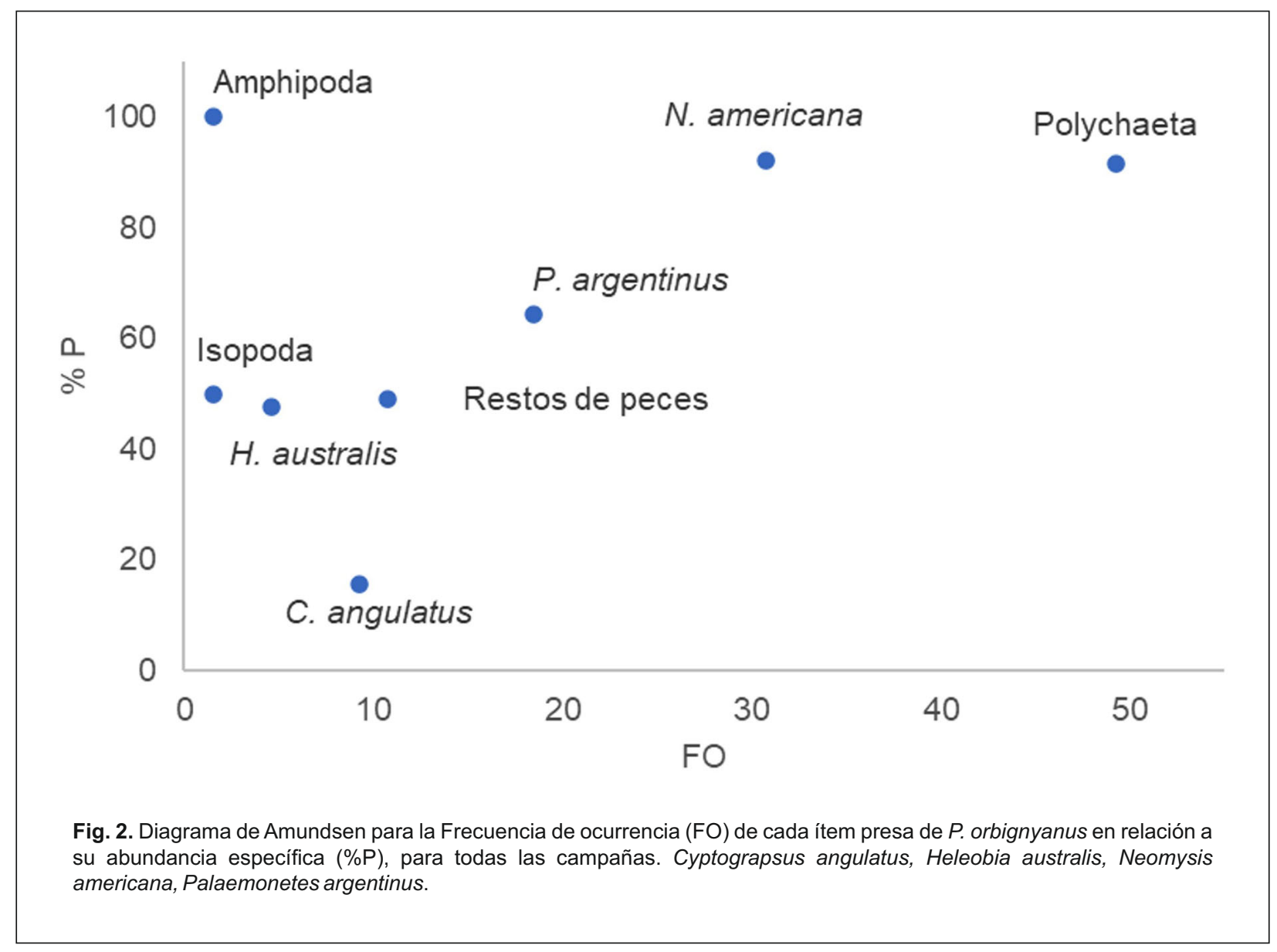

Bol. Soc. Zool. Uruguay (2a época). 2021. Vol. 30 (2): e30.2.9 ISSN 2393-6940

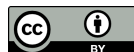




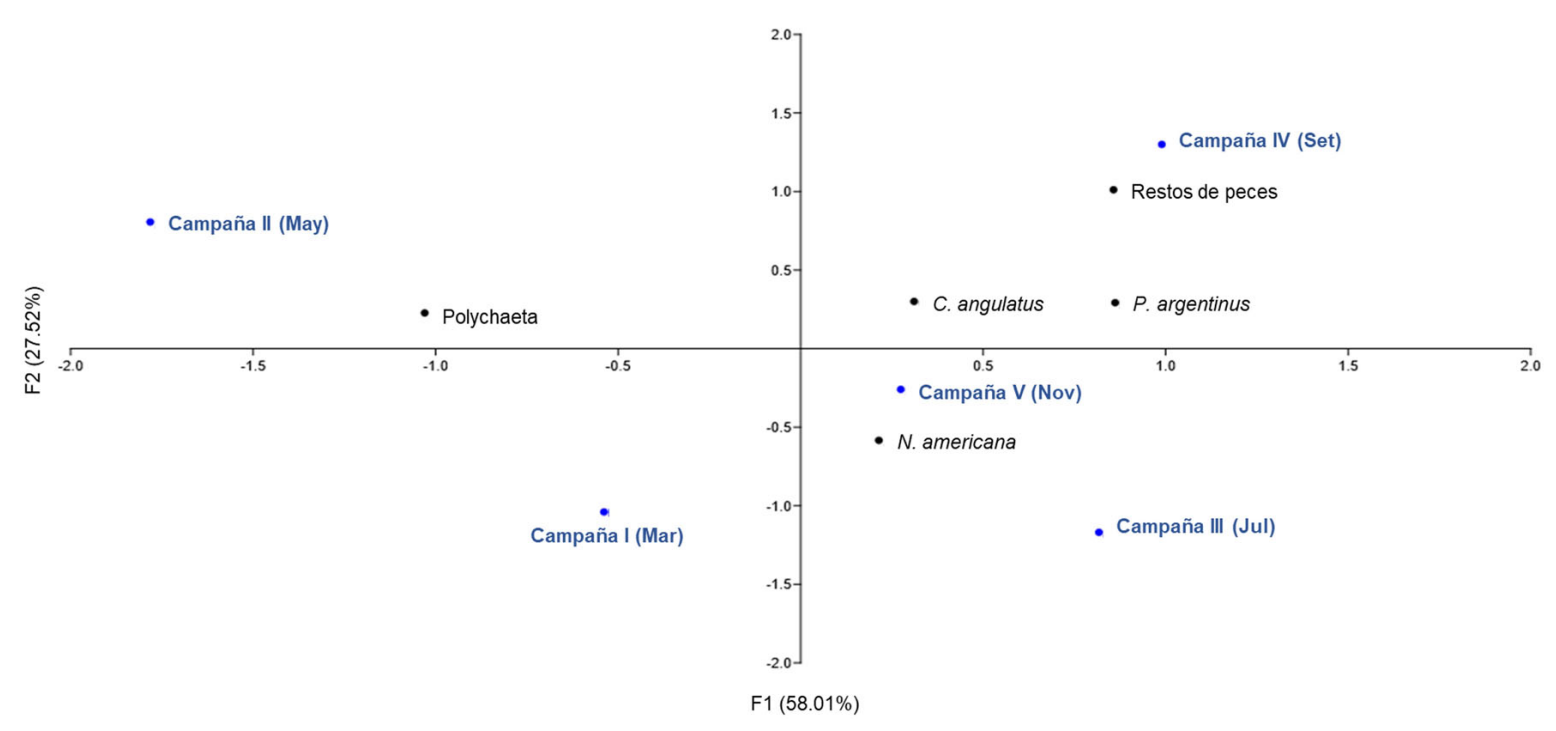

Fig. 3. Análisis de correspondencia entre los ítems presa y las diferentes campañas (marzo, mayo, julio, setiembre y noviembre). Cyptograpsus angulatus, Neomysis americana, Palaemonetes argentinus.

\section{RESULTADOS}

El $83 \%$ de los estómagos analizados presentaron contenido estomacal y el índice de vacuidad presentó su mayor valor en julio y el menor en setiembre. Se identificó un total de ocho ítems presa durante todas las campañas analizadas. En la campaña de marzo, los poliquetos representaron aproximadamente la mitad de la dieta (\%IRI: 52,6\%), mostrando el mayor valor en la siguiente campaña (\%IRI: 99,8\%, mayo) donde consumieron casi exclusivamente ese ítem presa, disminuyendo marcadamente en las siguientes campañas (Tabla 2). Los misidáceos (Neomysis americana), mostraron valores altos de \%IRI en las campañas de noviembre y julio (91,3 y 81,8 respectivamente) y menores en marzo $(47,9)$. Los camarones (Palaemonetes argentinus) representaron aproximadamente la mitad de los consumido en setiembre (Tabla 1). El diagrama de Amundsen que vincula la frecuencia de ocurrencia con a la Abundancia específica de cada ítem (Fig. 2), mostró que poliquetos y misidáceos fueron los ítems dominantes (alta frecuencia y abundancia) en la dieta de $P$. orbignyanus, seguidos por el ítem camarón. Sin embargo, los anfípodos presentaron altos niveles de abundancia específica, pero en una frecuencia baja.

Si bien al analizar la variación temporal de la dieta (en base al \%IRI) no se encontraron diferencias significativas $(p>0.05)$, entre las diferentes campañas, (test $F=4.34 \mathrm{E}-22, \mathrm{Gl}: 39$ ), el análisis de correspondencia de la frecuencia de ocurrencia entre los ítems presa y las diferentes campañas, explica el
$75 \%$ de la inercia en los ejes uno y dos. Los poliquetos se encuentran mayormente asociados a la campaña de mayo y los misidáceos a las campañas de noviembre y julio (Fig. 3).

EI LT promedio de los lenguados capturados aumenta de forma sostenida a lo largo del año, presentando diferencias significativas entre las campañas $\left(p<0,05\right.$; test $\left.\mathrm{K}-\mathrm{W}: \mathrm{H}_{\mathrm{c}}=26,0\right)$ (Tabla 2$)$. Las métricas del estado de condición de los peces (FC y \%Líp. T) mostraron tendencias distintas. Mientras el factor de condición presentó valores fluctuantes siendo en mayo y septiembre los valores más altos $\left(p<0,05\right.$; test $K-W: H_{c}=29,30$ ), las reservas lipídicas (\%Líp. T) tuvieron una disminución significativa $(p<0,05)$ en los peces obtenidos en la última campaña (noviembre) (ANOVA: $F=14,35$; gl=4), con valores por debajo de la mitad del contenido observado en las campañas anteriores. La humedad no presentó diferencias significativas $(p>0,05$; test K-W: $\left.\mathrm{H}_{\mathrm{c}}=0,4204\right)$.

En el perfil de ácidos grasos de los lípidos neutros del músculo con piel de los lenguados se observaron variaciones importantes a lo largo de las diferentes campañas (Tabla 3). En marzo (campaña I) se observó una mayor proporción de ácidos grasos poliinsaturados (PUFA, aprox. 47\%) y setiembre y noviembre la menor proporción (aprox. 40\%). A lo largo del periodo estudiado, se observó una disminución de la proporción de ácido eicosapentaenoico (EPA, 20:5n3) y una aumento de la de ácido araquidónico (AA, 20:4n-6), resultando en una relación AA/EPA muy superior en el perfil de ácidos grasos de noviembre. 
Tabla 2. Longitud total (LT), tenor lipídico en peso seco (\%Líp.T Ps), \% de humedad y factor de condición (FC) de los juveniles de lenguados capturados (valor \pm desviación estándar).

\begin{tabular}{lccccc} 
& Marzo & Mayo & Julio & Setiembre & Noviembre \\
\hline LT (cm) & $6,48 \pm 3,05 b$ & $8,53 \pm 2,21 b$ & $8,55 \pm 2,86 \mathrm{~b}$ & $10,02 \pm 2,28 \mathrm{ab}$ & $12,40 \pm 1,35 \mathrm{a}$ \\
\% Líp. T (Ps) & $5,68 \pm 1,64 \mathrm{a}$ & $5,34 \pm 1,17 \mathrm{a}$ & $4,91 \pm 1,08 \mathrm{a}$ & $6,29 \pm 0,98 \mathrm{a}$ & $2,56 \pm 0,48 \mathrm{~b}$ \\
\% Humedad & $76,90 \pm 3,66$ & $76,54 \pm 3,07$ & $72,93 \pm 10,53$ & $76,48 \pm 2,16$ & $76,80 \pm 2,55$ \\
FC & $0,94 \pm 0,08 \mathrm{~b}$ & $1,06 \pm 0,09 \mathrm{a}$ & $0,96 \pm 0,05 \mathrm{~b}$ & $1,07 \pm 0,10 \mathrm{a}$ & $0,96 \pm 0,09 \mathrm{~b}$ \\
\hline
\end{tabular}

\% Líp. T (Ps) y \% Humedad: marzo $n=4$; mayo $n=9$; julio $n=8$; setiembre $n=8$; noviembre $n=8$.

LT (cm) y FC: marzo $n=9$; mayo $n=31$; julio $n=14$; setiembre $n=16$; noviembre $n=13$.

Letra diferente en la misma fila indica diferencia significativa $(p<0,05)$.

Las proporciones más elevadas de ácido docosahexaenoico (DHA, 22:6n-3) se registraron en marzo y noviembre, aunque en noviembre se registró la mayor relación $\mathrm{DHA} / \mathrm{EPA}$.

El análisis de cluster muestra que los perfiles de ácidos grasos tienen una tendencia a agruparse entre las diferentes campañas (Fig. 4), los perfiles de mayo, julio y noviembre se ubicaron juntos en 3 clusters. Los perfiles de marzo quedaron dispersos pero en el mismo cluster, a diferencia de los perfiles de setiembre que fueron los que mostraron mayor dispersión, quedando incluso en clusters diferentes (Fig. 4).

\section{DISCUSIÓN}

En este estudio se observó que la dieta de los juveniles Paralichthys orbignyanus menores a un año en el arroyo Valizas estuvo compuesta mayormente por poliquetos y misidáceos ( $N$. americana). Resultados similares habían sido obtenidos en una laguna costera en Argentina (Mar Chiquita), donde Rivera et al., (2001), trabajando con el mismo rango de tamaño, encontraron que se alimenta principalmente de crustáceos (anfípodos y misidáceos) y poliquetos. Paralichthys orbignyanus, al igual que muchos lenguados, presenta un cambio ontogenético en su dieta (Link et al., 2005), consumiendo poliquetos y crustáceos en su etapa juvenil, pasando por una etapa intermedia en donde es reportado como carcinófago y piscívoro (Cazorla y Forte, 2005; Carnikián, 2006) finalizando en una alimentación piscívora en estado adulto (Norbis y Galli, 2004; Magnone et al., 2015b).

El alto porcentaje de estómagos con contenido (83\%) posiblemente refleja la buena disponibilidad de alimento en la zona de estudio, algo congruente con lo planteado por Gibson (1994) para los ambientes estuarinos. Al igual que en estudios anteriores realizados sobre la especie (Carneiro, 1995; Rivera et al., 2001), los mayores índices de vacuidad se encuentran en invierno y los menores en primavera.
La riqueza de ítems encontrada en los estómagos de juveniles de $P$. orbignyanus en el arroyo Valizas fue menor a la reportada en la laguna de Mar chiquita (Rivera et al., 2001). En dicho trabajo, al igual que en el nuestro, tampoco se registraron diferencias significativas en la composición de la dieta a lo largo del año. En nuestro caso en las campañas de setiembre y noviembre (primavera) fue cuando se reportó la mayor cantidad de ítems presa en los estómagos, a diferencia de los juveniles en la laguna de Mar Chiquita (Rivera et al., 2001) donde la diversidad fue mayor en otoño. La mayor cantidad de ítems encontrados en los estómagos de las campañas de primavera, podría deberse a una mayor diversidad de presas en el ambiente o podría vincularse con el aumento de tamaño de los peces, que les permitiría acceder a presas más grandes (peces óseos) (Link et al. 2005). Resulta esperable que a medida que van creciendo los juveniles se vayan tornando piscívoros (Norbis y Galli, 2004; Magnone et al., 2005a), dejando de lado las presas más pequeñas cuya obtención reditúe menos en términos energéticos. Para dilucidar estas interrogantes, sería conveniente llevar adelante estudios que consideren la evolución de la oferta de presas a lo largo del año.

Tradicionalmente, se estima el estado de los peces a través de las relaciones entre talla y peso con un índice conocido como Factor de Condición (FC) (Ricker, 1975). EI FC permite una estimación rápida de la condición del pez, aunque su capacidad para dilucidar procesos subyacentes es limitada (Arts y Kohler, 2009). Existen otros índices complementarios como el contenido de lípidos totales (\%Líp. T) que evalúan de forma más específica el estado de condión o nutricional de los peces (Sargent et al., 2002). Paralichthys orbignyanus almacena sus reservas lipídicas en el tejido subdérmico (Magnone et al., 2015b). En este trabajo los índices de condición (FC y \%Líp. T) mostraron tendencias diferentes en algunas campañas. Mientras el FC fluctuó suavemente en torno a un valor durante todo el año, el tenor lipídico 
Tabla 3. Principales AG de los lípidos neutros del músculo con piel de los lenguados capturados en las campañas I a $V$ (marzo, mayo, julio, setiembre y noviembre). AG expresados como porcentaje del total de AG área (valor \pm desviación estándar). SAFA: AG saturados; MUFA: AG monoinsaturados; PUFA: AG poliinsaturados; HUFA: AG altamente insaturados de cadena larga (>C20).

\begin{tabular}{cccccc} 
Ácido graso & Marzo & Mayo & Julio & Setiembre & Noviembre \\
\hline $16: 0$ & $19,4 \pm 1,6$ & $18,4 \pm 1,6$ & $20,1 \pm 1,2$ & $20,9 \pm 3,6$ & $23,3 \pm 1,8$ \\
$16: 1$ n-7 & $3,6 \pm 0,4$ & $5,1 \pm 1,5$ & $5,4 \pm 1,8$ & $6,2 \pm 1,7$ & $2,4 \pm 0,9$ \\
$18: 0$ & $5,4 \pm 0,3$ & $7,1 \pm 0,6$ & $6,0 \pm 0,8$ & $6,5 \pm 0,8$ & $9,8 \pm 1,2$ \\
$18: 1 n-9$ & $7,2 \pm 0,7$ & $7,2 \pm 1,1$ & $7,9 \pm 1,1$ & $9,2 \pm 1,7$ & $9,5 \pm 0,9$ \\
$18: 1 n-7$ & $3,8 \pm 0,5$ & $5,7 \pm 1,7$ & $4,4 \pm 0,9$ & $5,4 \pm 0,6$ & $3,6 \pm 0,6$ \\
$18: 2 n-6$ & $0,9 \pm 0,2$ & $1,3 \pm 0,1$ & $1,5 \pm 0,4$ & $1,6 \pm 0,2$ & $1,3 \pm 0,2$ \\
$18: 3 n-3$ & $2,6 \pm 3,0$ & $2,0 \pm 1,2$ & $2,6 \pm 2,2$ & $1,3 \pm 0,4$ & $0,5 \pm 0,2$ \\
$20: 1 n-9$ & $0,4 \pm 0,1$ & $0,8 \pm 0,1$ & $0,8 \pm 0,2$ & $0,7 \pm 0,1$ & $0,5 \pm 0,1$ \\
$20: 4 n-6$ & $3,1 \pm 0,4$ & $3,0 \pm 0,8$ & $3,4 \pm 0,9$ & $2,6 \pm 0,8$ & $4,7 \pm 0,5$ \\
$20: 5 n-3$ & $9,0 \pm 0,71$ & $1,4 \pm 1,11$ & $0,1 \pm 1,3$ & $7,8 \pm 2,5$ & $5,1 \pm 0,8$ \\
$22: 5 n-3$ & $4,0 \pm 0,6$ & $4,7 \pm 0,5$ & $4,6 \pm 0,8$ & $3,9 \pm 0,9$ & $3,7 \pm 0,5$ \\
$22: 6 n-3$ & $19,7 \pm 2,8$ & $9,2 \pm 6,71$ & $2,7 \pm 3,61$ & $3,1 \pm 4,32$ & $0,9 \pm 4,1$ \\
SAFA & $33,2 \pm 3,43$ & $1,2 \pm 1,33$ & $1,7 \pm 1,43$ & $3,5 \pm 4,73$ & $7,6 \pm 2,9$ \\
MUFA & $20,0 \pm 2,62$ & $5,9 \pm 4,02$ & $4,0 \pm 3,92$ & $7,2 \pm 3,81$ & $9,8 \pm 2,7$ \\
PUFA & $46,8 \pm 5,84$ & $3,0 \pm 3,44$ & $4,3 \pm 4,73$ & $9,3 \pm 8,14$ & $2,7 \pm 3,4$ \\
n-3 HUFA & $33,3 \pm 2,72$ & $6,3 \pm 5,82$ & $8,5 \pm 3,52$ & $5,7 \pm 6,53$ & $0,2 \pm 3,8$ \\
n-6/n-3 & $0,16 \pm 0,01$ & $0,31 \pm 0,06$ & $0,26 \pm 0,03$ & $0,25 \pm 0,06$ & $0,28 \pm 0,05$ \\
DHA/EPA & $2,22 \pm 0,46$ & $0,85 \pm 0,72$ & $1,30 \pm 0,51$ & $1,77 \pm 0,48$ & $4,26 \pm 1,38$ \\
AA/EPA & $0,35 \pm 0,07$ & $0,27 \pm 0,09$ & $0,34 \pm 0,13$ & $0,34 \pm 0,05$ & $0,92 \pm 0,07$ \\
\% Líp. T(Ps) & $5,68 \pm 1,64$ & $5,34 \pm 1,17$ & $4,91 \pm 1,08$ & $6,29 \pm 0,98$ & $2,56 \pm 0,48$
\end{tabular}

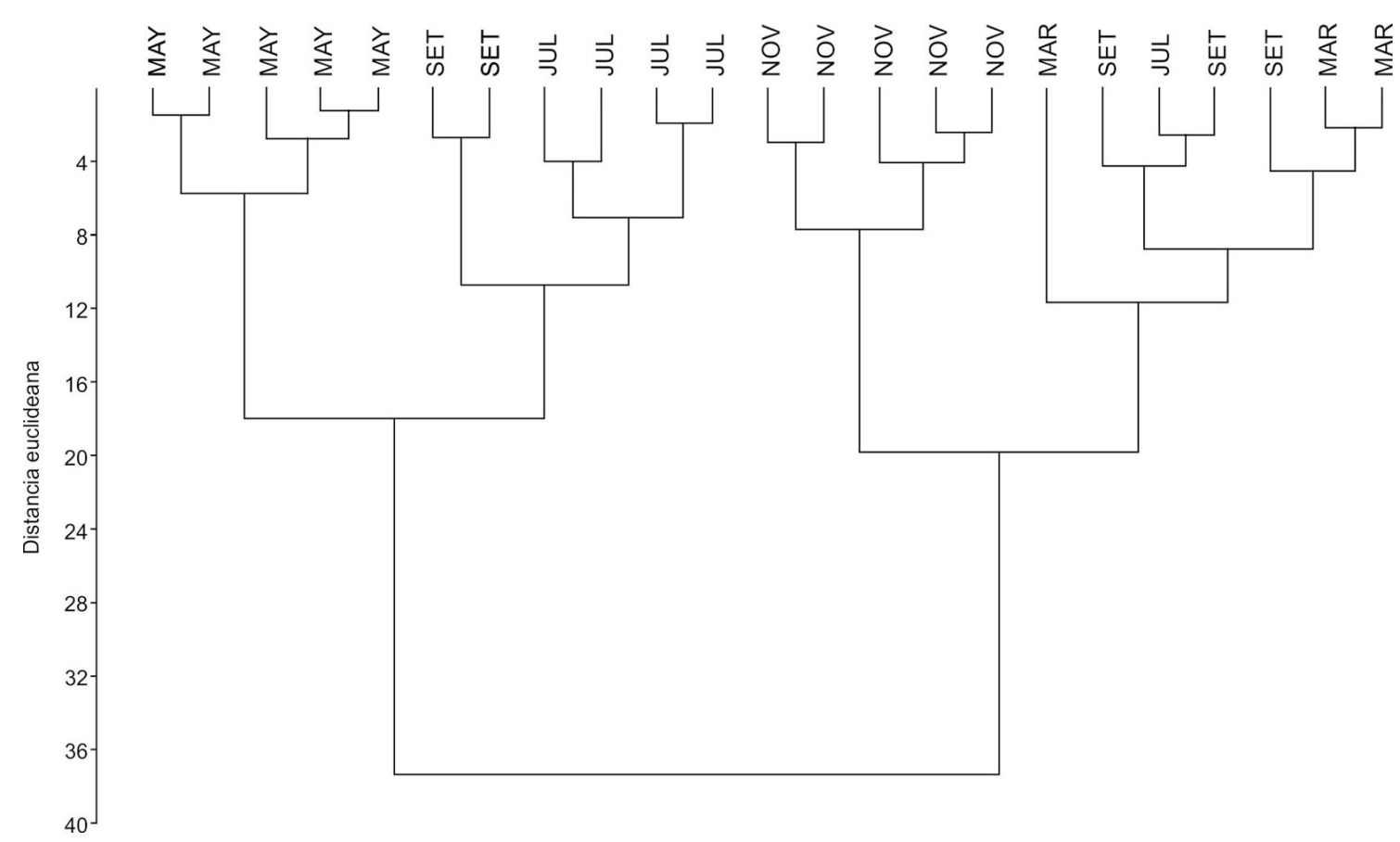

Fig. 4. Análisis de Cluster sobre los perfiles de AG de los ejemplares de Paralichthys orbignyanus capturados en el arroyo Valizas durante un ciclo anual, utilizando la totalidad de ácidos grasos identificados.

Bol. Soc. Zool. Uruguay (2a época). 2021. Vol. 30 (2): e30.2.9 ISSN 2393-6940 
(\%Líp. T) mostró una disminución muy marcada en la última campaña (noviembre). En la campaña de primavera (setiembre) ambos índices coinciden en los valores observados más altos ( $F C=1,07$, \%Líp. $\mathrm{T}=6,29$ ), pero en la campaña siguiente (noviembre) mientras el factor de condición presenta valores similares a las campñas anterioes, el tenor lípídico cae a valores significativamente menores $(2,56 \pm 0,48 \%)$. En esta campaña también se da un aumento significativo en la longitud total, sugiriendo que utilizan gran parte de las reservas lipídicas en el aumento de talla, lo que a su vez se ve reflejado en un FC inferior. A pesar de este consumo de reservas lipídicas, las proporciones de ácidos grasos como el AA y DHA (20:4n-6 y $22: 6 n-3$ respectivamente) se mantienen elevadas con respecto a las del EPA (22:5n-3), indicando la relevancia de estos ácidos tanto por su importancia fisiológica para los peces como por su eventual elevado contenido en las presas. Por otra parte, los peces además de movilizar prioritariamente para la ß-oxidación los MUFA y SAFA con respecto a los HUFA, se ha visto que particularmente el DHA es muy resistente a la degradación catabólica (Xu et al., 2020), resultando en una acumulación selectiva de este ácido graso en los tejidos.

El análisis exploratorio de cluster de los perfiles de ácidos grasos mostró una segregación temporal en la mayoría de sus perfiles (las campañas de mayo, julio y noviembre quedaron separadas en distintos clusters), lo que indicaría que los mismos van cambiando como reflejo de las variaciones estacionales de la dieta en las reservas lipídicas. Por otra parte, la similitud observada entrelos perfiles de marzo y septiembre, a pesar de las diferencias encontradas en la dieta, merece una reflexión acerca de las limtaciones de los análisis de conteido estomacal como método para estimar la misma, ya que con este abordaje se cuantifican principalmente las presas consumidas en la última ingesta y muchas veces se sobreestiman las presas con partes duras y se subestiman las de cuerpo blando (Jobling y Breiby, 1986; Bowen, 2000). Por este razón sería recomendable complementar estos estudios con otras metodologías como el análisis de isótopos estables o de ácidos grasos, abordajes que aportan una visión más integral de la dieta. En particular la especificidad biológica de los ácidos grasos y el hecho de que muchos de ellos no presentan mayores cambios en su estructura molecular luego de ser ingeridos e incorporados en los tejidos, los convierte en compuestos estratégicos para su uso como biomarcadores (Parrish et al., 2000).

Respecto a los perfiles de AG analizados de las distintas campañas, corresponde comentar que probablemente las diferencias encontradas en los niveles de DHA y EPA estén reflejando variaciones en la dieta, mientras que la importante presencia de ácido araquidónico (AA) observada en este trabajo probablemente se vincule a la retención característica que hacen los organismos de este ácido graso en ambientes estuarinos, que ya se ha reportado en otros trabajos (Koussoroplis et al., 2011; Magnone et al., $2015 b)$ y probablemente no esté reflejando directamente la dieta. A su vez el incremento en los niveles de EPA y la reducción de la relación DHA/EPA que se observaron en la segunda y tercera campaña se pueden corresponder con una ingesta de poliquetos, que se caracterizan por ser más ricos en EPA que en DHA (Magnone et al., 2015b; Santos et al., 2016). Por otra parte, el incremento de DHA y su relación con el EPA observado en las campañas siguientes se podría vincular a la ingesta de peces y misidáceos, que suelen presentar altos valores de estos AG (Magnone et al., 2015a y b; GrzeszczykKowalska et al., 2014).

De cualquier forma, es importante considerar que si bien estos abordajes que analizan AG esenciales y las relaciones entre ellos sin duda reflejan variaciones en la dieta y pueden ser indicadores cualitativos de la presencia de ciertos taxa en la misma (Budge, Iverson y Koopman, 2006), no dejan de ser indicativos, debido a su baja especificidad. Es recomendable para profundizar en el conocimiento de la dieta incluir en los estudios el tenor lipídico y el perfil de ácidos grasos de las presas mediante la aplicación de abordajes cuantitativos tipo QFASA (Iverson et al., 2004) que son capaces de cuantificar la dieta de predadores y muchas veces evidencian ítems presa difíciles de encontrar en el contenido estomacal.

\section{AGRADECIMIENTOS}

Los autores agradecen a los revisores anónimos por sus valiosos aportes durante la corrección del manuscrito.

\section{BIBLIOGRAFÍA}

Able, K. W. y Grothues, T. M. (2007). An approach to understanding habitat dynamics of flatfishes: advantages of biotelemetry. Journal of Sea Research,58(1), 1-7.

Acuña, A., Passadore, C. y Gimenez. L. (2010). Fish assemblage in a temperate estuary on the uruguayan coast: seasonal variation and environmental influence. Brazilian Journal of Oceanography, 58(4), 299-314.

Amundsen, P. A., Gabler, H. M. y Staldvik, F. J. (1996). A new approach to graphical analysis of feeding strategy from stomach contents data modification of the Costello (1990) method. Journal of fish biology, 48(4), 607-614.

Arts M., y Kohler C. (2009). Health and condition in fish: the influence of lipids on membrane competency and immune response. In: Kainz 
M., Brett M., Arts M. (eds) Lipids in Aquatic Ecosystems. Springer, New York, NY.

Budge, S., Iverson, S. y Koopman, H. (2006). Studying trophic ecology in marine ecosystems using fatty acids: a primer on analysis and interpretation. Marine Mammal Science, 22(4),759-801.

Bowen, W. D. (2000). Reconstruction of pinniped diets: accounting for complete digestion of otoliths and cephalopod beaks. Canadian Journal of Fisheries and Aquatic Sciences, 57(5), 898-905.

Carneiro, M. (1995). Reprodução e alimentação dos linguadosParalichthyspatagonicus e Paralichthysorbignyanus (Pleuronectiformes: Bothidae), no Rio Grande do Sul, Brasil. Dissertação de Mestrado. Fundação Universidade do Rio Grande do Sul, Brasil

Carnikian, A. (2006). Estudio de los hábitos alimenticios del lenguado (Paralichthys orbignyanus) en el estuario del Arroyo Pando. Tesina de Grado, Licenciatura en Ciencias Biológicas, Facultad de Ciencias, Universidad de la República, Uruguay, pp. 41.

Cazorla, A. L. (2005). On the age and growth of flounder Paralichthysorbignyanus (Jenyns, 1842) in Bahía Blanca Estuary, Argentina. Hydrobiologia, 537(1), 81-87.

Cazorla, A. L. y Forte, S. (2005). Food and feeding habits of flounder Paralichthys orbignyanus (Jenyns, 1842) in Bahía Blanca Estuary, Argentina. Hydrobiologia, 549(1), 251-257.

Christie, W. W. y Han, X. (2010). Lipid analysis. Isolation, separation, identification and lipidomic analysis. Oxford Cambridge Philadelphia New Delhi.

Cowan, J., Yañez-Arancibia, A., Sánchez-Gil, P. y Deegan, I. (2013). Estuarine Nekton. En: J. W. Day, B. C. Crump. y W. M. Kemp. (Eds). Estuarine Ecology (pp. 327-356). WuleyBlackwell.

Curtis, D., Galbraith, C, Smyth, J. y Thompson, D. (1985). Seasonal variations in prey selection by estuarine black-head gulls (Larus ridibundus). Estuarine, Coastal and Shelf Science, 21, 75-89.

Elliott M., Hemingway, K. L., Costello, M.J., Duhamel, S., Hostens, K., Labropoulou, M., ... Winkler, H. (2002). Links between Fish and Other Trophic Levels. En: M. Elliott y K. Hemingway (Eds). Fishes in Estuaries. (pp 124-216). Blackwell Publishing Ltd.

Espinosa, N. (2017). Rol de Neomysis americana (Crustacea: Mysinae) en los flujes de carbono en una laguna costera de la costa atlántica uruguaya. Tesis para optar por el título de Magíster en Geociencias. Facultad de Ciencias, Montevideo, Uruguay.
Folch, J., Lees, M. y Stanley, G. (1957). A simple method for isolation and purification of total lipids from animal tissues. The Journal of Biological Chemistry 226, 497-509.

Froneman, P. (2001). Seasonal changes in zooplancton biomass and grazing in a temperate estuary, South Africa. Estuarine, Coastal and Shelf Science, 52, 543-553.

Garrison, L. y Link, J. (2000). Dietary guild structure of the fish community in the northeast United States continental shelf ecosystem. Marine Ecology Progress Series, 202, 231-240.

Gibson, R. N. (1994). Impact of habitat quality and quantity on the recruitment of juvenile flatfishes. Netherlands Journal of Sea Research, 32(2), $191 \mathrm{v} 206$.

Grzeszczyk-Kowalska A., Chojnacki, J. C. Małgorzata R., Raczyńska, M. (2014) Biological characterization of Neomysis integer (Leach, 1815) from the Pomeranian Bay in 2006-2007. Journal of Ecological Engineering, 15(2), 40-52.

Hostens, K. y Mees, J. (1999). The mysid-feeding guild of demersal fishes in the brackish zone of the Westerschelde estuary. Journal of Fish Biology, 55, 704-719.

Hyslop, E. (1980). Stomach contents: a review of methods and their application. Journal of Fish Biology, 17(4), 411-429.

Iverson, S.J. (2009). Tracing aquatic food webs using fatty acids: from qualitative indicators to quantitative determination. En: M.T. Arts, M.T. Brett, M.J. Kainz, (Eds.) Lipids in Aquatic Ecosystems (pp 281-307). Springer, New York.

Iverson, S. J., Field, C., Bowen, W., Blanchard, W. (2004). Quantitative fatty acid signature analysis: a new method of estimating predator diets. Ecological Monographs, 74(2), 211-235.

Jobling, M. y Breiby, A. (1986). The use and abuse of fish otoliths in studies of feeding habits of marine piscivores. Sarsia, 71, 265-274.

Juaneda, P. y Rocquelin, G. (1985). Rapid and convenient separation of phospholipids and non phosphorus lipids from rat heart using silica cartridges. Lipids, 20, 40-41.

Koussoroplis, A., Bec, A., Perga, M., Koutrakis, E., Bourdier, G. y Desvilettes, C. (2011). Fatty acid transfer in the food web of a coastal Mediterranean lagoon: Evidence for high arachidonic acid retention in fish. Estuarine, Coastal and Shelf Science, 91, 450-461.

Laroche, J. L. (1982). Trophic patterns among larvae of five species of sculpins (Family: Cottidae) in a Maine estuary. Fishery Bulletin, 80(4),827-840.

Link, J., Fogarty, M. y Langton, R. (2005). The trophic ecology of flatfishes. En: R.N. Gibson, (Ed.). Flatfishes. (pp. 185-212). Blackwell Publishing, Oxford. 
Magnone, L., Bessonart, M., Gadea, J. y Salhi, M. (2015a). Trophic relationships in an estuarine environment: A quantitative fatty acid analysis signature approach. Estuarine, Coastal and Shelf Science, 166, 24-33.

Magnone, L., Bessonart, M., Rocamora, M., Gadea, J. y Salhi, M. (2015b). Diet estimation of Paralichthys orbignyanus in a coastal lagoon via quantitative fatty acid signature analysis. Journal of Experimental Marine Biology and Ecology, 462, 36-49.

Masdeu, M., Teixeira de Mello, F., Loureiro, M. y Arim, M. (2011). Feeding habits and morphometry of Iheringichthys labrosus (Luitken, 1874) in the Uruguay River (Uruguay). Neotropical Ichthyology, 9(3), 657-664.

McLusky, D. y Elliott, M. (2004a). Life in Estuaries En: D. McLusky y M. Elliott (Eds). The Estuarine Ecosystem: ecology, threats and management. (pp. 19-33). Oxford.

McLusky, D. y Elliott, M. (2004b). The secondary consumers: carnivores En: D. McLusky y M. Elliott (Eds). The Estuarine Ecosystem: ecology, threats and management. (pp. 73-91). Oxford.

Molinero, A. y Flos, R. (1992). Influence of season on the feeding habits of the common sole Solea solea. Marine Biology, 113(3), 499-507.

Norbis, W. y Galli, O. (2004). Hábitos de alimentación del lenguado Paralichthys orbignyanus (Valenciennes 1842) en una laguna costera somera del Atlántico Sur: Rocha, Uruguay. Ciencias Marinas, 30, 619-626.

Parrish, C., Abrajano, T., Budge, S., Helleur, R., Hudson, E., Pulchan, K. y Ramos, C. (2000). Lipid and phenolic biomarkers in marine ecosystems: analysis and applications. En: Wangersky, P. (Ed.). The Handbook of Environmental Chemistry, Part D, Marine Chemistry (pp.193-233). Springer, Berling, Heidelber.

Peterson, M. (2003). A conceptual view of environment-habitat-production linkages in tidal river estuaries. Reviews of Fisheries Science, 11, 291-313.

Piet, G., Pfisterer, A. y Rijnsdop, A. (1998). On factors structuring the flatfish assemblage in the southern North Sea. Netherlands Journal of Sea Research, 40, 143-152.

Pinkas, L., Oliphant, M. y Iverson, I. (1971). Food habits study of albacore, bluefin tuna, and bonito in California waters. Fish Bulletin, 152, 1-105.

Ricker, W. 1975. Computation and interpretation of biological statistics of fish populations. Bulletin Fisheries Research. Board of Canada, 191, 1382.

Rivera, A., García de la Rosa, S. y Díaz de Astarloa, J. (2001). Feeding ecology of flatfish juveniles (Pleuronectiformes) in Mar Chiquita coastal lagoon (Buenos Aires, Argentina). Estuaries, 24(6A), 917-125.

Salhi, M. y Bessonart, M. (2013). Growth, survival and fatty acid composition of Rhamdia quelen (Quoy and Gaimard, 1824) larvae fed on artificial diet alone or in combination with Artemia nauplii. Aquaculture Research, 44, 41-79.

Santos, A., Granada, L., Baptista, T., Anjos, C., Simões, T., Tecelão, C., ... Pombo, A. (2006). Effect of three diets on the growth and fatty acid profile of the common ragworm Hedistedi versicolor (O.F. Muiller, 1776). Aquaculture, 465, 37-42.

Sargent, J. R., Tocher, D. R. y Bell, J. G. (2002). The Lipids. En: Halver, J. E., Hardy, R. W. (Eds). Fish Nutrition. (pp. 181-257). Academic Press, San Diego.

Vaz-Ferreira, R. (1969). Peces del Uruguay. Aljanati, D., Benedeto, M. Marsillo H. (Eds). Colección Nuestra Tierra, 23, 72.

Williams, D. y Williams, N. (1998). Seasonal variation, export dynamics and consumption of freshwater invertebrates in an estuarine environment. Estuarine, Coastal and Shelf Science, 46, 393-410.

Wouters, N. y Cabral, H. (2009). Are flatfish nursery grounds richer in benthic prey?.Estuarine, Coastal and Shelf Science, 83, 613-620.

Xu, H., Turchini, G.M., Francis, D.S., Liang, M., Mock, T.S., Rombenso, A. y Ai, Q. (2020). Are fish what they eat? A fatty acid's perspective. Progress in Lipid Research, 80, 101064.

Editor de Sección: Franco Teixeira de Mello 